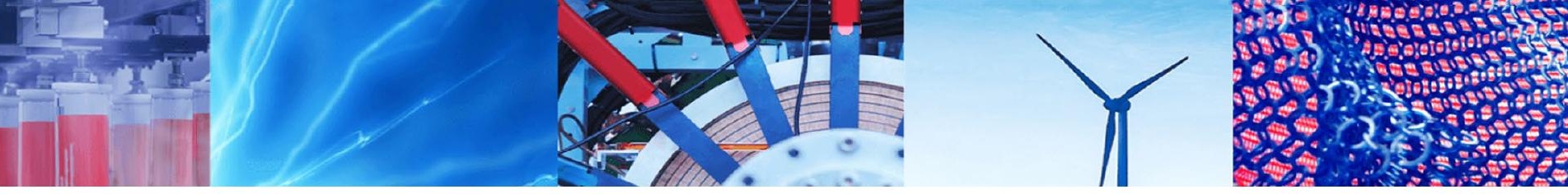

Research Article

\title{
Tensor sparse PCA and face recognition: a novel approach
}

\author{
Loc $\operatorname{Tran}^{1} \cdot$ Linh $\operatorname{Tran}^{2}$ (1) $\cdot$ Trang Hoang $^{2} \cdot$ Bao Bui $^{2}$
}

Received: 11 March 2020 / Accepted: 30 May 2020 / Published online: 15 June 2020

(c) Springer Nature Switzerland AG 2020

\begin{abstract}
Face recognition is the important field in machine learning and pattern recognition research area. It has a lot of applications in military, finance, public security, to name a few. In this paper, the combination of the tensor sparse PCA with the nearest-neighbor method (and with the kernel ridge regression method) will be proposed and applied to the face dataset. Experimental results show that the combination of the tensor sparse PCA with any classification system does not always reach the best accuracy performance measures. However, the accuracy of the combination of the sparse PCA method and one specific classification system is always better than the accuracy of the combination of the PCA method and one specific classification system and is always better than the accuracy of the classification system itself.
\end{abstract}

Keywords Tensor $\cdot$ Sparse PCA $\cdot$ Kernel ridge regression $\cdot$ Face recognition $\cdot$ Nearest-neighbor

\section{Introduction}

Face recognition is the important field in machine learning and pattern recognition research area. It has a lot of applications in military, finance, public security, to name a few. Computer scientists have worked in face recognition research area for almost three decades. In the early 1990, the Eigenface [1] technique has been employed to recognize faces and it can be considered the first approach used to recognize faces. The Eigenface [1] used the principle component analysis (PCA) which is one of the most popular dimensional reduction methods $[2,3]$ to reduce the dimensions of the faces. This PCA technique can also be used in other pattern recognition problems such as speech recognition $[4,5]$. After using PCA to reduce the dimensions of the faces, the Eigenface use nearest-neighbor method [6] to recognize or to classify faces.

To classify the faces, a graph (i.e., kernel) which is the natural model of relationship between faces can also be employed. In this model, the nodes represent faces. The edges represent for the possible interactions between nodes. Then, machine learning methods such as support vector machine [7], kernel ridge regression [8], artificial neural networks [9] or the graph-based semi-supervised learning methods [10-12] can be applied to this graph to classify or to recognize the faces. The artificial neural networks, support vector machine, and kernel ridge regression are supervised learning methods. The graph-based semi-supervised learning methods are the semi-supervised learning methods. Please note that the kernel ridge regression method is the simplest form of the support vector machine method.

In the last two decades, the SVM learning method has successfully been applied to some specific classification tasks such as digit recognition, text classification, and protein function prediction and face recognition problem [7]. However, the kernel ridge regression method (i.e., the simplest form of the SVM method) has not been applied to any practical applications. Hence, in this paper, in addition to the nearest-neighbor method, we will also use the kernel ridge regression method applied to the face recognition problem.

\footnotetext{
$\triangle$ Linh Tran, linhtran@hcmut.edu.vn; LocTran, tran0398@umn.edu; Trang Hoang, hoangtrang@hcmut.edu.vn; Bao Bui, buiquocbao@ hcmut.edu.vn | ${ }^{1}$ Laboratoire CHArt EA4004, EPHE-PSL University, Paris, France. ${ }^{2}$ Ho Chi Minh City University of Technology - VNU HCM, Ho Chi Minh City, Vietnam.
} 
Last but not least, in this literature review part, we would like to discuss a little bit about deep learning approach in general and about the deep convolution neural network in specific. In recent years, the deep convolution neural network can be considered the current state-of-the-art method for image classification problems [13-19] and it has been applied to solve a lot of big data classification problems in various fields such as natural language processing field and computer vision field. However, in our paper, the dataset that we used contains only hundreds of face images. Thus, it is not appropriate and suitable to employ the deep convolutional neural network to classify such a tiny image dataset. In the other words, we just employ the classic nearest neighbor method and the classic kernel ridge regression method to classify our images.

Next, we will introduce the principle component analysis. Principle component analysis (i.e., PCA) is one of the most popular dimensionality reduction techniques [2]. It has several applications in many areas such as pattern recognition, computer vision, statistics, and data analysis. It employs the eigenvectors of the covariance matrix of the feature data to project on a lower dimensional subspace. This will lead to the reduction in noises and redundant features in the data and the low time complexity of the nearest-neighbor and the kernel ridge regression approach solving face recognition problem.

However, the PCA has two major disadvantages which are the lack of sparsity of the loading vectors and each principle component is the linear combination of all variables. From data analysis viewpoint, sparsity is necessary for reduced computational time and better generalization performance. From modeling viewpoint, although the interpretability of linear combinations is usually easy for low-dimensional data, it could become much harder when the number of variables becomes large. To overcome this hardness and to introduce sparsity, many methods have been proposed such as [20-23]. In this paper, we will introduce new approach for sparse PCA using alternating direction method of multipliers (i.e., ADMM method) [24]. Then, we will try to combine the sparse PCA dimensional reduction method with the nearest-neighbor method (and with the kernel ridge regression method) and apply these combinations to the face recognition problem. This work, to the best of our knowledge, has not been investigated.

Last but not least, we will also develop the novel tensor sparse PCA method and apply this dimensional reduction method to the face database (i.e., tensor) in order to reduce the dimensions of the face database. Then, we will apply the nearest-neighbor method or the kernel ridge regression method to recognize the transformed faces.

We will organize the paper as follows: Sect. 2 will present the alternating direction method of multipliers.
Section 3 will derive the sparse PCA method using the ADMM method in detail. Section 4 will present the sparse PCA algorithm. Section 5 will present the detailed version of the tensor sparse PCA algorithm. In Sect. 6, we will apply the combination of tensor sparse PCA algorithm with the nearest-neighbor algorithm and with the kernel ridge regression algorithm to faces in the dataset available from [25]. Section 7 will conclude this paper and discuss the future directions of researches of this face recognition problem.

\section{Alternating direction method of multipliers}

In this section, we will introduce the alternating direction method of multipliers. The detailed information about the alternating direction method of multipliers can be found in [24]. First, assume that we want to solve the following problem

minimize $f(x)+g(z)$

subject to $A x+B z=c$

with variables $x \in R^{n}$ and $z \in R^{m}$, where $A \in R^{p * n}, B \in R^{p * m}$

Next, we will form the augmented Lagrangian

$L_{\rho}(x, z, y)=f(x)+g(z)+y^{\top}(A x+B z-c)$

$$
+\frac{p}{2}\|A x+B z-c\|_{2}^{2}
$$

Finally, $x^{k+1}, z^{k+1}$ and $y^{k+1}$ can be solved as the followings

$x^{k+1}=\operatorname{argmin}_{x} L_{\rho}\left(x, z^{k}, y^{k}\right)$

$z^{k+1}=\operatorname{argmin}_{z} L_{\rho}\left(x^{k+1}, z, y^{k}\right)$

$y^{k+1}=y^{k}+\rho\left(A x^{k+1}+B z^{k+1}-c\right)$,

where $\rho>0$ is the positive penalty parameter.

\section{Sparse principle component analysis derivation}

Assume that we are given the data matrix $D \in R^{n+p}(n$ is the number of samples and $p$ is the number of features). Next, we will formulate our sparse PCA problem. This problem is in fact the following optimization problem.

$\operatorname{minimize}_{x, z}-\|\tilde{D} x\|_{2}^{2}+\lambda\|z\|_{1}$

such that $x=z$ and $\|z\| \leq 1$.

Our objective is to find the sparse vector $x$. Please note that 
$\tilde{D}=\left|\begin{array}{c}d_{1}-\mu \\ d_{2}-\mu \\ \cdot \\ \cdot \\ \cdot \\ d_{n}-\mu\end{array}\right|$,

where $\mu=\frac{1}{n} \sum_{i=1}^{n} d_{i}$ be the mean vector of all row vectors $d_{1}, d_{2}, \ldots, d_{n}$ of $D$.

First, the augmented Lagrangian of the above optimization problem can be derived as the following:

$$
\begin{aligned}
L_{p}(x, z, y)= & -\|\tilde{D} x\|_{2}^{2}+\lambda\|z\|_{1}+y^{T}(x-z) \\
& +\frac{\rho}{2}\|x-z\|_{2}^{2}
\end{aligned}
$$

Then, $x^{k+1}, z^{k+1}$, and $y^{k+1}$ can be solved as the followings:

$x^{k+1}=\operatorname{argmin}_{x} L_{\rho}\left(x, z^{k}, y^{k}\right)$

Hence,

$$
\begin{aligned}
\frac{\mathrm{d} x^{k+1}}{\mathrm{~d} x} & =\frac{\mathrm{d}}{\mathrm{d} x}\left(-\|\tilde{D} x\|_{2}^{2}+y^{k^{T}}\left(x-z^{k}\right)+\frac{\rho}{2}\left\|x-z^{k}\right\|_{2}^{2}\right) \\
& =\frac{\mathrm{d}}{\mathrm{d} x}\left(-x^{T} \tilde{D}^{T} \tilde{D} x+y^{k^{T}}\left(x-z^{k}\right)+\frac{\rho}{2}\left\|x-z^{k}\right\|_{2}^{2}\right) \\
& =-2 \tilde{D}^{T} \tilde{D} x+y^{k}+\rho\left(x-z^{k}\right)
\end{aligned}
$$

Next, we solve

$\frac{\mathrm{d} x^{k+1}}{\mathrm{~d} x}=0 \Leftrightarrow\left(-2 \tilde{D}^{T} \tilde{D}+\rho l\right) x=-y^{k}+\rho z^{k}$

Thus,

$x^{k+1}=\left(-2 \tilde{D}^{T} \tilde{D}+\rho l\right)^{-1}\left(-y^{k}+\rho z^{k}\right)$

Next, we have

$$
z^{k+1}=\operatorname{argmin}_{z} L_{\rho}\left(x^{k+1}, z, y^{k}\right)
$$

Hence,

$$
\begin{aligned}
\frac{\mathrm{d} z^{k+1}}{\mathrm{~d} z} & =\frac{\mathrm{d}}{\mathrm{d} z}\left(\lambda\|z\|_{1}+y^{k^{T}}\left(x^{k+1}-z\right)+\frac{\rho}{2}\left\|x^{k+1}-z\right\|_{2}^{2}\right) \\
& =\lambda \xi-y^{k}+\rho\left(x^{k+1}-z\right)(-1) \\
& =\lambda \xi-y^{k}+\rho\left(z-x^{k+1}\right)
\end{aligned}
$$

where

$$
\xi_{i}= \begin{cases}1 & \text { if } z_{i}>0 \\ {[-1,1]} & \text { if } z_{i}=0 \\ -1 & \text { if } z_{i}<0\end{cases}
$$

Solve $\frac{d z^{k+1}}{d z}=0$, we have

$z_{i}^{k+1}=x_{i}^{k+1}+\frac{1}{\rho} y_{i}^{k}-\frac{\lambda}{\rho} \xi_{i}$

if $z_{i}^{k+1}>0, \xi_{i}=1$, then

$x_{i}^{k+1}+\frac{1}{\rho} y_{i}^{k}-\frac{\lambda}{\rho}>0 \Rightarrow x_{i}^{k+1}+\frac{1}{\rho} y_{i}^{k}>\frac{\lambda}{\rho}$

if $z_{i}^{k+1}<0, \xi_{i}=-1$, then

$x_{i}^{k+1}+\frac{1}{\rho} y_{i}^{k}+\frac{\lambda}{\rho}<0 \Rightarrow x_{i}^{k+1}+\frac{1}{\rho} y_{i}^{k}<-\frac{\lambda}{\rho}$

if $z_{i}^{k+1}=0$, then

$-\frac{\lambda}{\rho} \leq x_{i}^{k+1}+\frac{1}{\rho} y_{i}^{k} \leq \frac{\lambda}{\rho}$

Thus,

$z_{i}=\operatorname{sign}\left(x_{i}^{k+1}+\frac{1}{\rho} y_{i}^{k}\right) \max \left(\left|x_{i}^{k+1}+\frac{1}{\rho} y_{i}^{k}\right|-\frac{\lambda}{\rho}, 0\right)$

Finally, we have

$$
y^{k+1}=y^{k}+\rho\left(x^{k+1}-z^{k+1}\right)
$$

\section{Sparse principle component analysis algorithm}

In this section, we will present the sparse PCA algorithm 


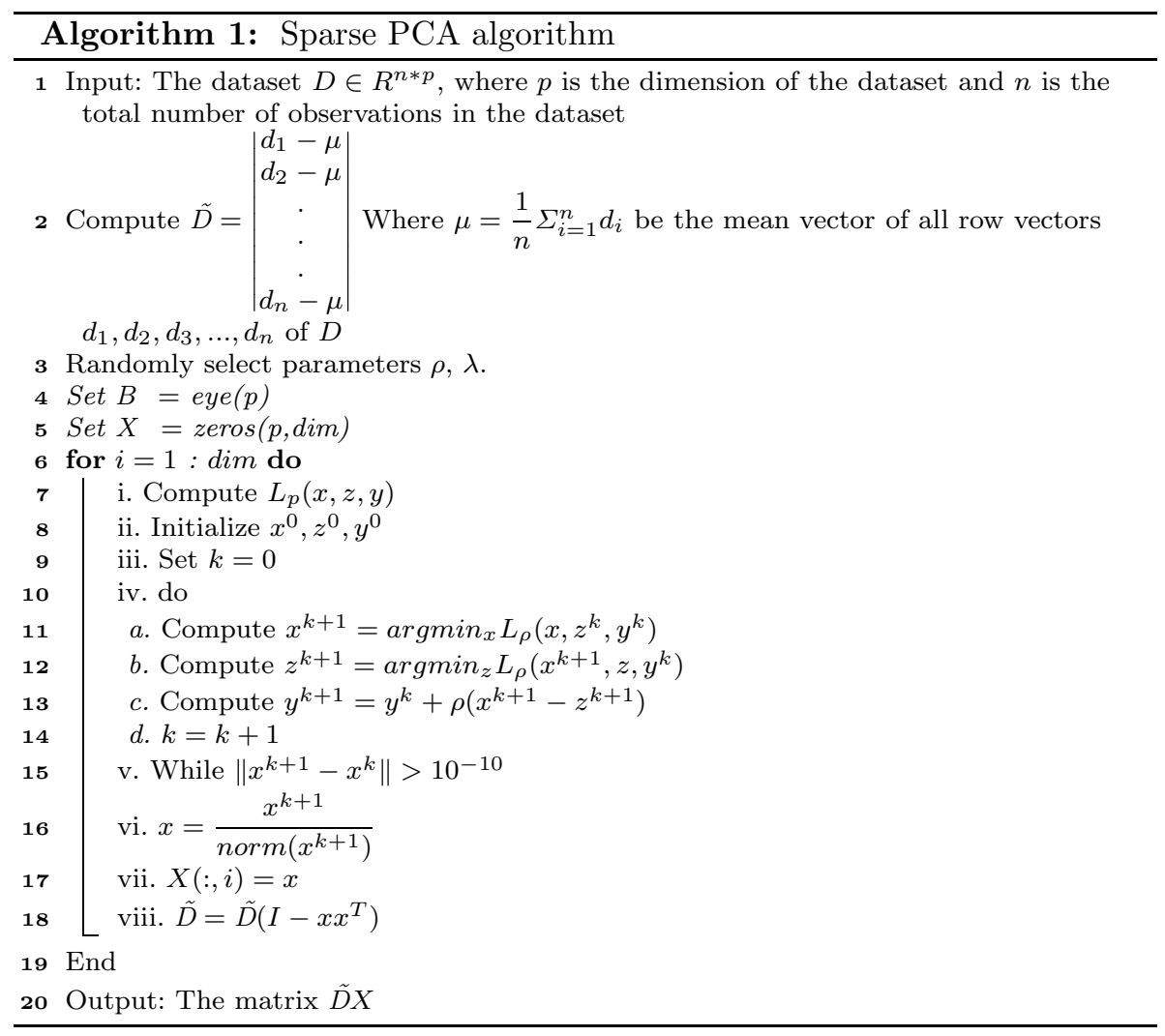

\section{Tensor sparse principle component analysis algorithm}

In this section, we will present tensor sparse PCA method.

Suppose $Y$ is a three mode tensor with dimensionalities $N_{1}, N_{2}$, and $N_{3}$, where $N_{1}$ is the height of the image, $N_{2}$ is the width of the image, $N_{3}=n_{1}+\cdots+n_{P}$ is the total number of images in the dataset and $P$ is the total number of people in the dataset. Then, the first mode unfolding of $Y$ is written as $Y_{(1)}$ and is the matrix $N_{2} N_{3} * N_{1}$. The second mode unfolding of $Y$ is written as $Y_{(2)}$ and is the matrix $N_{1} N_{3} * N_{2}$. The third mode unfolding of $Y$ is written as $Y_{(3)}$ and is the matrix $N_{1} N_{2} * N_{3}$.

The refolding is the reverse operation of the unfolding operation. Thus, the tensor sparse PCA can be represented as follows

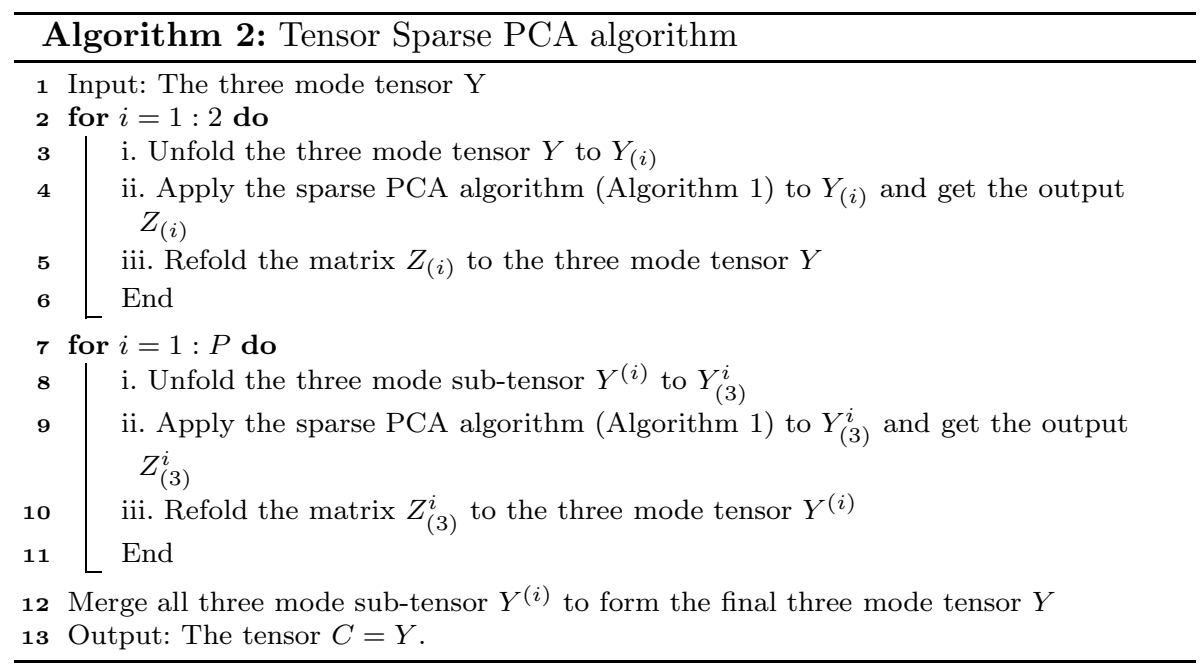




\section{Experiments and results}

In this paper, we use the images from the Yale dataset [25] to test our approaches. This dataset contains 165 grayscale images in GIF format of 15 people. There are 11 images per people, one per different facial expression: center-light, with glasses, happy, left-light, etc. Then, the set of 120 face samples recorded of 15 different people (8 face samples per people) are used for training. Then, another set of 45 face samples of these people ( 3 faces samples per people) are used for testing the accuracy measure. The description of this dataset and this dataset itself are available from [25]. Each face sample (i.e., the image) is the $R^{(32 * 32)}$ matrix. Then, we will merge all rows of the face sample (i.e., the matrix) sequentially from the first row to the last row into a single big row which is the $R^{(1 * 1024)}$ row vector (i.e., $32 * 32=1024$ ). These row vectors will be used as the feature vectors of the nearest-neighbor method and the kernel ridge regression method.

Next, the PCA and the sparse PCA algorithms will be applied to face samples in the training set and the testing set to reduce the dimensions of the face samples in the dataset. Please note that the PCA algorithm is also the dimensional reduction method. Information about PCA algorithm can be found in [1]. After applying the PCA and the sparse PCA algorithms to the face samples in the training set and the testing set, the number of dimensions of these transformed feature vectors of the Yale face dataset will be 200,300,400,500, and 600 . Then, the nearestneighbor method and the kernel ridge regression method will be applied to these new transformed feature vectors.

At the very beginning, each face sample is the $R^{(32 * 32)}$ matrix. There are 120 face samples in the training set. So, the training set is in fact the $R^{(32 * 32 * 120)}$ tensor. There are 45 face samples in the testing set. Hence, the testing set is in fact the $R^{(32 * 32 * 45)}$ tensor. Then, we apply the tensor sparse PCA algorithm to the training set and the testing set to reduce the dimensions of the face dataset. The tensor sparse PCA algorithm is described clearly in Sect. 5, algorithm 2 . Now, the training set is the $R^{(25 * 25 * 75)}$ tensor and the testing set is the $R^{(25 * 25 * 45)}$ tensor. Then, we will merge all rows of the face sample (i.e., the new matrix) into a single big row which is the $R^{(1 * 625)}$ row vector. Finally, the nearest-neighbor method and the kernel ridge regression method will be applied to the new transformed dataset.
Table 1 Accuracies of the nearest-neighbor method, the combination of PCA method and the nearest-neighbor method, the combination of sparse PCA method and the nearest-neighbor method, and the combination of tensor sparse PCA method and the nearest-neighbor method (\%)

Table 2 Accuracies of the kernel ridge regression method, the combination of PCA method and the kernel ridge regression method, the combination of sparse PCA method and the kernel ridge regression method, and the combination of tensor sparse PCA method and the kernel ridge regression method

\begin{tabular}{ll}
\hline The nearest-neighbor method & 90.81 \\
\hline PCA $(d=200)+$ The nearest-neighbor method & 91.11 \\
PCA $(d=300)+$ The nearest-neighbor method & 91.11 \\
PCA $(d=400)+$ The nearest-neighbor method & 91.11 \\
PCA $(d=500)+$ The nearest-neighbor method & 91.11 \\
PCA $(d=600)+$ The nearest-neighbor method & 91.11 \\
Sparse PCA $(d=200)+$ The nearest-neighbor method & $91.70(89.02 \pm 2.42)$ \\
Sparse PCA $(d=300)+$ The nearest-neighbor method & $91.70(90.40 \pm 1.31)$ \\
Sparse PCA $(d=400)+$ The nearest-neighbor method & $91.41(88.23 \pm 2.25)$ \\
Sparse PCA $(d=500)+$ The nearest-neighbor method & $91.70(87.57 \pm 3.18)$ \\
Sparse PCA $(d=600)+$ The nearest-neighbor method & $91.11(89.02 \pm 1.13)$ \\
Tensor sparse PCA + The nearest-neighbor method & $\mathbf{9 2}(89.59 \pm 1.42)$ \\
\hline
\end{tabular}

Bold indicate those methods reach the highest accuracy

\begin{tabular}{|c|c|}
\hline The kernel ridge regression method & 95.85 \\
\hline PCA $(d=200)+$ The kernel ridge regression method & 96.15 \\
\hline PCA $(d=300)+$ The kernel ridge regression method & 96.15 \\
\hline PCA $(d=400)+$ The kernel ridge regression method & 96.15 \\
\hline PCA $(d=500)+$ The kernel ridge regression method & 96.15 \\
\hline PCA $(d=600)+$ The kernel ridge regression method & 96.15 \\
\hline Sparse PCA $(d=200)+$ The kernel ridge regression method & $96.15(92.84 \pm 3.19)$ \\
\hline Sparse PCA $(d=300)+$ The kernel ridge regression method & $96.44(91.01 \pm 4.02)$ \\
\hline Sparse PCA $(d=400)+$ The kernel ridge regression method & $96.44(93.10 \pm 2.27)$ \\
\hline Sparse PCA $(d=500)+$ The kernel ridge regression method & $96.15(91.90 \pm 3.14)$ \\
\hline Sparse PCA $(d=600)+$ The kernel ridge regression method & $96.74(94.42 \pm 1.31)$ \\
\hline Tensor sparse PCA + The kernel ridge regression method & $87.85(85.63 \pm 1.18)$ \\
\hline
\end{tabular}

Bold indicate those methods reach the highest accuracy 
In this section, we experiment with the above nearestneighbor method and kernel ridge regression method in terms of accuracy measure. The accuracy measure $Q$ is given as follows:

$Q=\frac{\text { True Positive }+ \text { True Negative }}{\text { True Positive + True Negative + False Positive + False Negative }}$

All experiments were implemented in Matlab 6.5 on virtual machine. For the nearest neighbor method, the number of neighbors that is used is 23 . We run each method 20 times, and the best accuracy performance measure of each method is recorded. In recent years, the deep convolutional neural network can be considered the current state-of-the-art method for image classification problem. However, it just has been applied to big data only. In our experiments, our used dataset just contains only hundreds of face images. Thus, it is not appropriate and not suitable to use the deep convolutional neural network in this paper, due to lack of time and lack of space. The accuracy performance measures of the above proposed methods are given in the following Tables 1 and 2 .

From the above tables, we recognize that the combination of the tensor sparse PCA method and one specific classification system does not always reach the best accuracy performance measure. This accuracy depends on the classification system. However, the accuracy of the combination of the sparse PCA method and one specific classification system is always better than the accuracy of the combination of the PCA method and one specific classification system and is always better than the accuracy of the classification system itself. The reasons are given in Sect. 1 .

The time complexities of the tensor sparse PCA and the sparse PCA is not recorded here since these two methods employ the ADMM optimization technique which is the very fast optimization technique. Information about the time complexity of ADMM optimization technique can be found in [24].

\section{Conclusion}

In this paper, the detailed versions of the sparse PCA method and the tensor sparse PCA method have been proposed. The experimental results show that the combination of the tensor sparse PCA with any specific classification system does not always reach the best accuracy performance measure. However, the accuracy of the combination of the sparse PCA method and one specific classification system is always better than the accuracy of the combination of the PCA method and one specific classification system and is always better than the accuracy of the classification system itself.
In the future, we will test the accuracies of the combination of the tensor sparse PCA method with a lot of classification systems, for, e.g., the SVM method or deep neural network methods.

In this paper, we use the alternating direction method of multipliers (i.e., the ADMM method) to solve the sparse PCA problem. In the near future, we will use the other optimization methods such as the proximal gradient method to solve the sparse PCA problem. Then, we will compare the accuracies and the run time complexities among these optimization techniques. To the best of our knowledge, this work has not been investigated up to now.

Acknowledgements This research is funded by Ho Chi Minh City University of Technology - VNU-HCM under Grant No. T-DDT-2018-79.

\section{Compliance with ethical standards}

Conflict of interest On behalf of all authors, the corresponding author states that there is no conflict of interest.

\section{References}

1. Turk M, Pentland A (1991) Eigenfaces for recognition. J Cogn Neurosci 3(1):71-86

2. Tran $\mathrm{LH}$, Tran $\mathrm{LH}$, Trang $\mathrm{H}$ (2015) Combinatorial and random walk hypergraph laplacian eigenmaps. Int J Mach Learn Comput 5(6):462

3. Tran L, Mai A, Quan T, Tran L (2018) Weighted un-normalized hypergraph Laplacian eigenmaps for classification problems. Int J Adv Soft Comput Appl 10(3):190-205

4. Trang H, Loc TH, Nam HBH (2014) Proposed combination of PCA and MFCC feature extraction in speech recognition system. In: 2014 international conference on advanced technologies for communications (ATC 2014). IEEE

5. Tran LH, Tran LH (2018) The combination of sparse principle component analysis and kernel ridge regression methods applied to speech recognition problem. Int J Adv Soft Comput Appl 10(2)

6. Zhang Z (2016) Introduction to machine learning: k-nearest neighbors. Ann Transl Med 4(11):218

7. Scholkopf B, Smola AJ (2001) Learning with kernels: support vector machines, regularization, optimization, and beyond. MIT Press, Cambridge

8. Trang H, Tran L (2014) Kernel ridge regression method applied to speech recognition problem: a novel approach. In: 2014 International conference on advanced technologies for communications (ATC 2014). IEEE

9. Zurada JM (1992) Introduction to artificial neural systems, vol 8. West Publishing Company, St. Paul

10. Tran L (2012) Application of three graph Laplacian based semisupervised learning methods to protein function prediction problem. arXiv preprint arXiv:1211.4289

11. Tran L, Tran L (2017) The un-normalized graph $p$-Laplacian based semi-supervised learning method and speech recognition problem. Int J Adv Soft Comput Appl 9(1):1-13

12. Trang H, Tran LH (2014) Graph based semi-supervised learning methods applied to speech recognition problem. In: 
International conference on nature of computation and communication. Springer, Cham

13. Altuntaş Y, Cömert Z, Kocamaz AF (2019) Identification of haploid and diploid maize seeds using convolutional neural networks and a transfer learning approach. Comput Electron Agric 163:104874

14. Gavali P, Banu JS (2020) Bird species identification using deep learning on GPU platform. In: 2020 International conference on emerging trends in information technology and engineering (ic-ETITE). IEEE

15. Krizhevsky A, Sutskever I, Hinton EG (2012) Imagenet classification with deep convolutional neural networks. In: Advances in Neural Information Processing Systems 25 (NIPS 2012)

16. Traore BB, Kamsu-Foguem B, Tangara F (2018) Deep convolution neural network for image recognition. Ecol Inform 48:257-268

17. Rawat W, Wang Z (2017) Deep convolutional neural networks for image classification: a comprehensive review. Neural Comput 29(9):2352-2449

18. El-Rahiem BA et al (2019) An efficient deep convolutional neural network for visual image classification. In: International conference on advanced machine learning technologies and applications. Springer, Cham
19. Huan E-Y, Wen G-H, Zhang S-J, Li D-Y, Hu Y, Chang T-Y, Wang Q, Huang B-L (2017) Deep convolutional neural networks for classifying body constitution based on face image. Computat Math Methods Med. https://doi.org/10.1155/2017/9846707

20. Hausman RE (1982) Constrained multivariate analysis. Stud Manag Sci 19:137-151

21. Vines SK (2000) Simple principal components. J R Stat Soc Ser C (Appl Stat) 49(4):441-451

22. Jolliffe IT, Trendafilov NT, Uddin M (2003) A modified principal component technique based on the LASSO. J Comput Graph Stat 12(3):531-547

23. Zou H, Hastie T, Tibshirani R (2006) Sparse principal component analysis. J Comput Graph Stat 15(2):265-286

24. Boyd $S$ et al (2011) Distributed optimization and statistical learning via the alternating direction method of multipliers. Found Trends Mach Learn 3(1):1-122

25. http://www.cad.zju.edu.cn/home/dengcai/Data/FaceData.html

Publisher's Note Springer Nature remains neutral with regard to jurisdictional claims in published maps and institutional affiliations. 